\title{
COMMUNITY-BASED EARLY WARNING AND ADAPTIVE RESPONSE SYSTEM (EWARS) FOR MOSQUITO BORNE DISEASES: AN OPEN SOURCE/OPEN COMMUNITY APPROACH
}

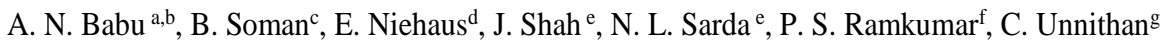 \\ ${ }^{a}$ Center for Advancement of Global Health, Kochi, Kerala, India (ajitnbabu@ gmail.com) \\ ${ }^{\mathrm{b}}$ Saint Louis University, St. Louis MO, USA \\ ${ }^{\mathrm{c}}$ Achutha Menon Centre for Health Science Studies, SCTIMST, Thiruvananthapuram, Kerala, India \\ ${ }^{\mathrm{d}}$ University of Koblenz-Landau, Landau, Germany - \\ ${ }^{\mathrm{e}}$ GISE Advanced Research Lab, Indian Institute of Technology-Bombay, Mumbai, India \\ ${ }^{\mathrm{f}}$ Applied Cognition Systems, Bengaluru, Karnataka, India \\ ${ }^{g}$ Deakin University, Melbourne, Australia
}

\begin{abstract}
:
A variety of studies around the world have evaluated the use of remote sensing with and without GIS in communicable diseases. The ongoing Ebola epidemic has highlighted the risks that can arise for the global community from rapidly spreading diseases which may outpace attempts at control and eradication. This paper presents an approach to the development, deployment, validation and wide-spread adoption of a GIS-based temporo-spatial decision support system which is being collaboratively developed in open source/open community mode by an international group that came together under UN auspices. The group believes in an open source/open community approach to make the fruits of knowledge as widely accessible as possible. A core initiative of the groups is the EWARS project. It proposes to strengthen existing public health systems by the development and validation a model for a community based surveillance and response system which will initially address mosquito borne diseases in the developing world. At present mathematical modeling to support EWARS is at an advanced state, and it planned to embark on a pilot project
\end{abstract}

\section{INTRODUCTION}

This paper presents an approach to the development, deployment, validation and wide-spread adoption of a GISbased temporo-spatial decision support system which is being collaboratively developed in open source/open community mode by an international group that came together under UN auspices. The EWARS project proposes to strengthen existing public health systems by the development and validation a model for a community based surveillance and response system

\section{EWARS OVERVIEW}

\subsection{Collaborative group}

The EWARS project involves an international group of scientists and academicians collaborating with governments, initially under the broad umbrella of a United Nations Action Team (AT 6) that was formed by the United Nations Office for Outer Space Affairs (UNOOSA) for fostering the use of spacebased technology in public health. The five-year term for AT 6 concluded in 2012, but the initiatives started during AT 6 are ongoing under the auspices of the AT 6 Follow Up Initiative (AT6FUI) with continuing involvement of UNOOSA and WHO, Geneva. The EWARS project is one of the major projects of the AT6FUI. Key constituents include representatives from India and Germany. The group has used innovative approaches like virtual meetings to minimize costs which will initially address mosquito borne diseases in the developing world. In pursuit of this objective, an informatics based temporo-spatial decision support system is under development which will ultimately use existing data collected by the public health system to generate dynamic risk maps that can provide early warning to the possibility of mosquito borne epidemics. An EWARS (Early Warning and Automated Response System) platform directed to control of mosquito borne diseases of global importance like dengue, malaria and chikungunya will be considered in this project and permit maximum participation of stakeholders from around the globe.

\subsection{Core principles}

- Open source, open community model. All project derived tools and resources can be freely used by anyone.

- Collaboration of equals - participants should be prepared not only to take what they need but also to contribute something useful to the project

- The project will be deemed successful only when it has actually been adopted by the public health 
system, and is making a positive difference to the actual delivery of services which improves health outcomes

- Optimization of resources will be done wherever possible to get the maximal

\subsection{Rationale}

A variety of studies around the world have evaluated the use of remote sensing with and without GIS in communicable diseases. The ongoing Ebola epidemic has highlighted the risks that can arise for the global community from rapidly spreading diseases which may outpace attempts at control and eradication. It is widely acknowledged that GIS and remote sensing based tools may have significant value in For example, investigators studying Japanese Encephalitis (JE) in the NorthEast state of Assam in India (Dhiman 2013), Filariasis in India (Sabesan 2000), Cholera in Bangladesh (Alam 2006) and Malaria in varying locations around India (Saxena 2009), have all shown promising results. These findings however have not yet led to the adoption of temporo-spatial decision support systems by the existing public health systems in the country. In addition, these efforts did not attempt to support a targeted response to the problem. The proposed project therefore plans to address these shortcomings by developing and validating such a system through a participatory action research methodology, with an initial focus on mosquito-borne disorders of significance both in India and other developing countries, specifically dengue, malaria and chikungunya. In the future, EWARS can be expanded to include other diseases of interest.

\section{STUDY DESIGN}

Action research and living labs methodology for community driven data collection and validation will be evaluated. A systematic approach will be followed as outlined:

1. Risk mapping would be carried out using the known variables as well as additional factors that may be relevant from a public health perspective.

2. RS data would be collected about "fixed" objects like roads, houses, stable water bodies, agriculture and vegetation distributions etc. GIS data has already been collected for over 35,000 households covering a population of about 131,000 .

3. An automated risk score would then be developed such that the geographic area where the greatest public health threat is deemed present would be given the highest rating.

4. Resources to deal with the identified problems would be automatically recommended for allocation based on the database of existing resources that would be compiled as part of the system with an adaptive "fuzzy-logic" methodology [Classical mathematical logic operates with statements and rules that can be evaluated as "true" or "false" (1 or 0 respectively). Rule based systems in the public health sector have to deal with statements that have a degree of truth ranging between 0 and 1. Fuzzy logic methodology is an innovative and scientifically sound approach to implement decision support in this complex environment] (Niehaus 2009).

5. The areas under surveillance would then be reviewed using GIS and field data on a defined schedule to determine if the identified problems have been resolved or reduced in severity by intervention of the public health system.

6. Risk scoring would change automatically and dynamically based on updated system inputs and resources would be automatically recommended for allocation in real-time based on the revised list of highest threats.

7. Using as far as possible existing avenues such as the Rural Employment Generation Scheme, a cadre of volunteers would be identified in collaboration with the panchayat bodies and entrusted with the responsibility of maintaining local sanitation and environmental hygiene in the community. They would act as the frontline workers that initiative prompt local action in response to the decision support system.

8. Health functionaries in the existing system (including doctors, field health workers etc) would be encouraged to provide health education and control strategies whenever necessary.

9. The performance of the system would be monitored on an ongoing basis, and improvements made such that accuracy and capabilities of the model are enhanced with the passage of time.

10. The EWARS system will be constructed in such a way that it can be scalable for eventual use in multiple ways and in a variety of disease conditions including non-communicable diseases.

11. Findings from the project will be disseminated through meetings, conferences, UN and other governmental entities and the media.

\section{POLICY IMPLICATIONS OF THE PROJECT}

The proposed system is a comprehensive temporo-spatial Decision Support System. It supports health service administration in risk evaluation and allocation of resources based on risk scores. Feasibility and acceptance of EWARS at the level of administration, users and general populace will be systematically studied using both quantitative and qualitative methods incorporating elements of action research methodology and community-led development theory along 
with more traditional approaches such as models and case studies. Also developed will be methods for optimizing the collection and review of data, as well as processes for enhancing data quality.

This project if successful has the potential to transform public health service delivery in developing countries by supporting scientifically sound, timely and cost-effective decisions which can greatly improve the health of our communities. The open community model also allows the possibility of enhancing global collaboration and information sharing for a greater social good. The current Ebola epidemic in Africa offers a good example

\section{CURRENT STATUS OF THE PROJECT}

- AMCHSS has collected data on 35,461 households covering a population of 131,095 individuals, as well as on relevant topographical features, roads, public buildings and so on. This offers significant value to the further development of the geospatial database required for which findings from the AMCHSS micro-study area will be crucial.

- A trained cadre of women volunteers have also been established in the micro-pilot area. They are local residents who are well-versed with the area and easily accepted by the other residents in the area.

- Mathematical modeling and development of customizable user interfaces for PC's and handheld devices ready for pilot testing (Germany)

- Collaboration building, collection of preliminary data, identification of sources for existing relevant data accomplished. Pilot study proposed to commence in 2015 (India)

- Last AT6FUI virtual meeting was held in August 2014, "hosted" by the Sree Chitra Institute in Trivandrum

\section{POSITIVE OUTCOMES}

1. At minimal expenditure, a widely dispersed group of international collaborators following an open community approach have been able to develop the primary components of an open source DSS for early warning and adaptive response.

2. A viable methodology has been arrived at to permit global collaboration between like-minded people at minimal expense. An innovative "virtual" meeting methodology was piloted in 2012 and refined each year since then for the group's annual meeting. In our view, one of the elements that hamper global collaboration amongst individuals who want to contribute to a social cause or project is the effort and expense that may be involved. To circumvent this to the maximal extent, and as a practical application of our philosophy of resource optimization, we presently do not hold traditional conference type gatherings where attendees are expected to physically attend. Rather through use of low-bandwidth multi-point videoconferencing and online repositories that are easily accessible like YouTube, we follow a process where meeting "presenters" post videos of their talks well ahead of the "meeting" date. Then, during the virtual meeting, a specific timeline and agenda are followed, centering around interactive discussion of the posted presentations and related brainstorming. This not only saves on expenses related to travel and stay, but also allows an asynchronous approach to information sharing where members can spend as much or as little time on a given presentation, have the opportunity to do additional reading or related information gathering and ultimately get the maximum return on the time and effort invested. In between meetings, communication and collaboration continues through electronic means.

3. Over time, the efforts of the group have created increased awareness at various levels of Indian governance about the benefits of a GIS based informatics system.

\section{CHALLENGES}

1. While a significant amount has been accomplished without external funding, the key step of initiating a pilot will clearly require funds. Attempts are in progress for securing funding through traditional means, but there are consequent uncertainties. The voluntary contributions of well-meaning individuals while capable of leading to significant accomplishment overall, may not be in a position to

2. A second alternative of partnering directly with the government has also not shown a clear way forward. Despite interest from individual government functionaries, there appears to be a general hesitancy in embracing the unknown and possibly facing adverse consequences due to unforeseen impact from the use of such a system.

3. The rather egalitarian collaboration model without a clearly defined administrative hierarchy can sometimes lead to a lack of cohesion or steady progress towards desired goals.

\section{CONCLUSION}

An EWARS platform based on an open source/open community approach can provide a cost-effective means of leveraging the benefits of geospatial data married to advanced mathematical modeling to enable epidemic prediction and response. This paper will share key findings and approaches that can be useful to others who may wish to adopt a similar approach to tackling the grave problem of vector-borne diseases in low-resource settings. Also presented are our experiences with using innovative ICT approaches towards global collaboration with the outcome of facilitating collegial interaction at minimal expense and loss of time on travel and logistics. 


\section{REFERENCES}

1: Dhiman S, Rabha B, Talukdar PK, Das NG, Yadav K, Baruah I, Singh L, Veer V. 2013. DDT \& deltamethrin resistance status of known Japanese encephalitis vectors in Assam, India. Indian J Med Res. Dec;138(6):988-94.

2: Sabesan S, Raju HK, Srividya A, Das PK. 2006. Delimitation of lymphatic filariasis transmission risk areas: a geo-environmental approach. Filaria J. Nov 9;5:12.

3: Alam M, Hasan NA, Sadique A, Bhuiyan NA, Ahmed KU, Nusrin S, Nair GB, Siddique AK, Sack RB, Sack DA, Huq A, Colwell RR. 2006. Seasonal cholera caused by Vibrio cholerae serogroups $\mathrm{O} 1$ and $\mathrm{O} 139$ in the coastal aquatic environment of Bangladesh.Appl Environ Microbiol. Jun;72(6):4096-104.

4: Saxena R, Nagpal BN, Srivastava A, Gupta SK, Dash AP. 2009. Application of spatial technology in malaria research \& control: some new insights. Indian J Med Res.Aug;130(2):12532.

5. Niehaus E., Herselman M., Babu AN. 2009. Principles of Neuroempiricism and generalization of network topology for health service delivery. Sep 15. Indian Journal of Medical Informatics. [Online] 4:1 